\title{
Geometric Approach to Hole Segmentation and Hole Closing in 3D Volumetric Objects
}

\author{
Marcin Janaszewski ${ }^{1}$, Michel Couprie $^{2}$, and Laurent Babout ${ }^{1}$ \\ 1 Computer Engineering Department,Technical University of Łódź, \\ Stefanowskiego 18/22, 90-924 Łódź, Poland \\ \{janasz, lbabout\}@kis.p.lodz.pl \\ 2 Université Paris-Est, LIGM, Equipe A3SI, ESIEE, \\ Cité DESCARTES BP 9993162 Noisy le Grand CEDEX, France \\ coupriem@esiee.fr
}

\begin{abstract}
Hole segmentation (or hole filling) and hole closing in 3D volumetric objects, visualised in tomographic images, has many potential applications in material science and medicine. On the other hand there is no algorithm for hole segmentation in $3 \mathrm{D}$ volumetric objects as from the topological point of view a hole is not a $3 \mathrm{D}$ set. Therefore in the paper the authors present a new, geometrical approach to hole closing and hole filling in volumetric objects. Moreover an original and efficient, flexible algorithm of hole filling for volumetric objects is presented. The algorithm has been extensively tested on various types of 3D images. Some results of the algorithm application in material science for crack propagation analysis are also presented. The paper also includes discussion of the obtained results and the algorithm properties.
\end{abstract}

\section{Introduction}

From the topological point of view the presence of a hole in an object is detected whenever there is a closed path which cannot be transformed into a single point by a sequence of elementary local deformations inside the object [7]. For example, a sphere has no hole, a solid torus has one hole and a hollow torus has two holes. Unfortunately, from a topological point of view a hole is not a subset of $3 \mathrm{D}$ space so it can not be segmented or filled. On the other hand, there is strong practical need to treat holes as $3 \mathrm{D}$ subsets. In material science hole segmentation can contribute to the quantification of damage phenomena that can help to understand and further optimise the resistance of the material to damage [26]. Other possible medical applications may consist in filling small noisy holes in 3D tomographs of human organs. Such a hole filling is especially desired in analysis of 3D computer tomography images of bronchial tubes where noisy holes in a bronchial tree significantly complicate automatic quantitative analysis [8]. Therefore, taking into account the topological point of view we propose a geometrical approach, which considers the notion of the thickness of an object and interpolates the thickness in the corresponding hole filling volume.

E. Bayro-Corrochano and J.-O. Eklundh (Eds.): CIARP 2009, LNCS 5856, pp. 25:-262, 2009.

(C) Springer-Verlag Berlin Heidelberg 2009 


\section{Basic Notions}

In this section, we recall some basic topological notions for binary images. A more extensive review is provided in $7 / 3$.

We denote by $\mathbb{Z}$ the set of integers, $\mathbb{N}_{+}$set of positive integers. Let $E=\mathbb{Z}^{3}$. Informally, a simple point $p$ of a discrete object $X \subset E$ is a point which is "inessential" to the topology of $X$. In other words, we can remove the point $p$ from $X$ without "changing the topology of $X$ ".

Skipping some technical details, let $A(x, X)$ be the set of points of $X \backslash\{x\}$ lying in a neighborhood of $x$, and let $A b(x, X)$ be the set of points of the complementary of $X$ (background) lying in a neighborhood of $x$. Then, $T(x, X)$ (resp. $T b(x, X))$ is the number of connected components of $A(x, X)$ (resp. $A b(x, X))$. A point $x \in X$ is simple for $X$ if and only if $T(x, X)=T b(x, X)=1$. Also, if a point $x \in X$ is such that $T b(x, X)=1$, then removing $x$ from $X$ does not create a new hole.

Let $X$ be any finite subset of $E$. The subset $Y$ of $E$ is a homotopic thinning of $X$ if $Y=X$ or if $Y$ may be obtained from $X$ by iterative deletion of simple points. We say that $Y$ is an ultimate homotopic skeleton of $X$ if $Y$ is a homotopic thinning of $X$ and if there is no simple point for $Y$.

Let $x \in E, r \in \mathbb{N}_{+}$, we denote by $B_{r}(x)$ the ball of (squared) radius $r$ centred on $x$, defined by $B_{r}(x)=\left\{y \in E, d^{2}(x, y)<r\right\}$, where $d^{2}(x, y)$ is a squared Euclidean distance for any $x, y \in E$.

A ball $B_{r}(x) \subseteq X \subseteq E$ is maximal for $X$ if it is not strictly included in any other ball included in $X$.

The medial axis of $X$, denoted by $\mathrm{MA}(X)$, is the set of the centres of all the maximal balls for $X$.

The thickness of an object $X$ in point $x$ belonging to $\mathrm{MA}(X)$ or to a skeleton of $X$ is defined as a radius of the maximal ball centred in $x$.

\section{Hole Notion from Geometrical Point of View}

In our approach we consider holes from a geometrical point of view but we base our consideration on the topological definition of a hole presented in Sect. 1 .

Intuitively, if we fill a hole in an object, the filling volume may be treated as a representation of the hole. In other words, the thickness (see Sect. 2) of the hole filling volume at its skeletal voxels should be equal to the thickness of the object at the skeletal voxels which are near the hole. Moreover, the shape of the boundary surface of a filling volume should fit the shape of the corresponding object's hole in the same way as two pieces of a puzzle match each other. The example of a hole filling volume for a frame is presented in Fig. 1. Unfortunately, it is very difficult to precisely define, in mathematical manner a hole filling volume. Taking into account the above comments, we are only able to propose two conditions which should be fulfilled by any segmented hole filling volume:

1. A hole filling volume should close a corresponding hole. 
(a)

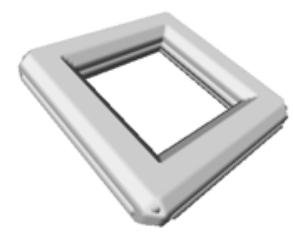

(b)

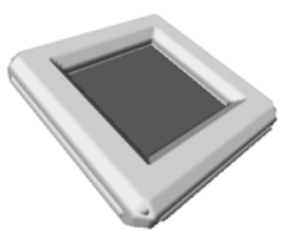

(c)

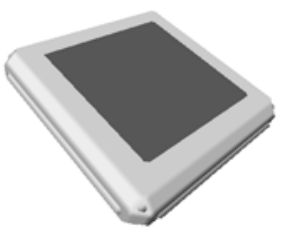

(d)

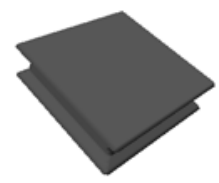

Fig. 1. An example of hole filling volume and hole closing patch for a frame: (a) isosurface of a frame; (b) frame with the hole closed; (c) frame with the hole filled. Hole filling volume is indicated with dark grey colour; (d) isosurface of the frame hole filling volume.

2. The thickness of a hole filling volume at its skeletal voxels should be equal to the thickness of the object on its medial axis (or skeletal) voxels which are close the hole.

\section{Modified Hole Closing Algorithm}

One of the main steps of hole filling algorithm (HFA) consists in application of a modified version of the hole closing algorithm (HCA) proposed by Aktouf et al. 11. The original version of the algorithm is linear in time and space complexity and takes as an input volumetric objects and closes all holes in these objects with one voxel thick patches. The pseudocode of the algorithm can be presented as follows:

\section{HCA ( Input $X$, Output $Y$ )}

Generate a full cuboid $Y$ which contains $X$

Repeat until no point to delete:

Select a point $p$ of $Y \backslash X$ which is at the greatest distance from $X$ and such that $T b(p, Y)=1$

$Y:=Y \backslash p$

Result: $Y$

An example of the algorithm result when applied to a 3D frame (Fig. 1(a))is presented in Fig. 1(b). It is worth mentioning the difference between hole filling and hole closing. The hole closing volume represented in dark grey colour in Fig. 1(b) is one voxel thick, independently on the thickness of the corresponding input object while the hole filling volume (see Fig. 1(c)) exactly matches the thickness of the corresponding object. More formal and extensive description of the algorithm can be found in 1 .

The most important drawback of HCA, from the hole filling point of view, is that the shape of a hole closing patch may be significantly influenced by irrelevant branches which are close to the hole. Such a situation is presented in Fig. 2 In our approach HCA takes as an input a skeleton of a 3D object. The skeleton is one voxel thick so the object presented in Fig. 2(a) is a good example 
(a)

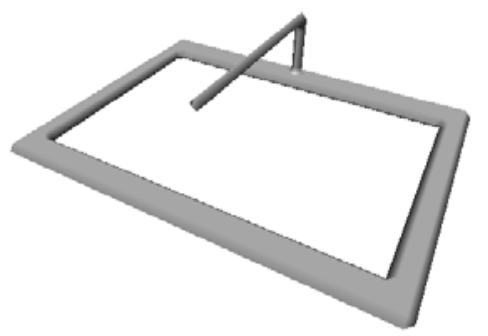

(c)

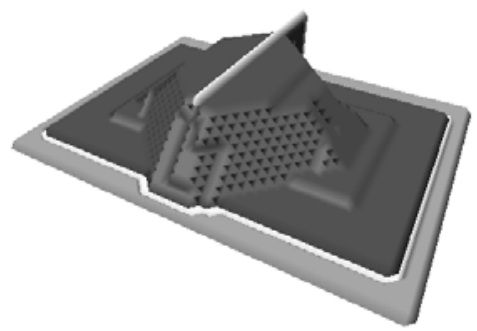

(e)

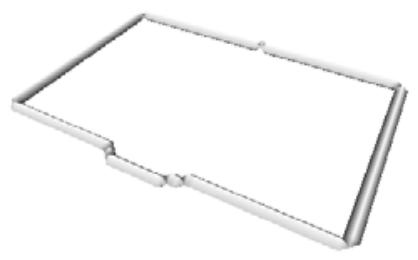

(b)

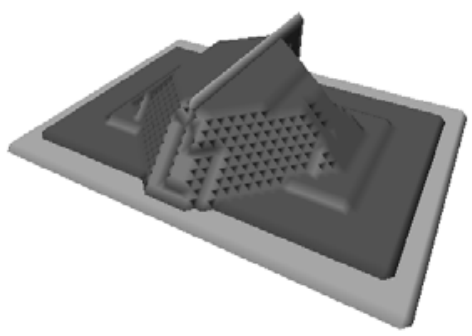

(d)

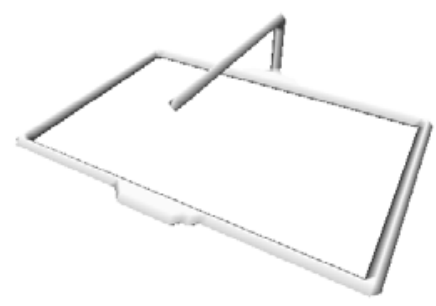

(f)

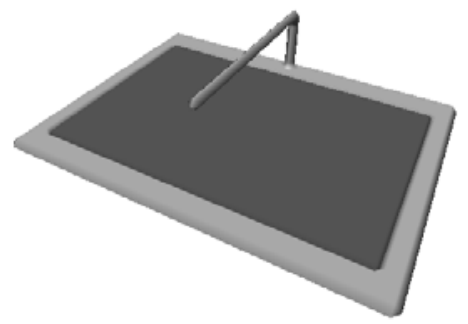

Fig. 2. Example result of each step of $\mathrm{HCA}+$ : (a) an isosurface of an input object; (b) the result of HCA. Notice that, the hole closing patch (dark grey colour) goes up to the branch situated over the hole; (c) the result of geodesic dilation of the patch over the input object. The intersection of dilated patch and input object, called hole contour (white colour); (d) visualisation of the hole contour, where one can see all its details (e) the result of ultimate homotopic skeletonisation algorithm applied to the hole contour. Note that, the branch has been deleted and topology of the hole contour has been preserved; (f) result of HCA, applied to the hole contour superimposed to the input object. Note that, the hole closing patch (dark grey colour) is not influenced by the branch.

of an input object for HCA. There is one big hole in the middle of the object and a thin branch above. Figure 2(b) presents the result of HCA. The hole closing patch, which is represented with dark grey colour, goes up to the branch, so it does not correspond to the "geometry of the hole", which leads to wrong hole filling. In this case we expect that the hole closing patch is flat as the object around the hole is flat. To overcome this problem we propose a 4-step method based on the HCA. The first step of the method consists in application of HCA. 
As a result we obtain an object with the hole closed but the hole closing path is influenced by the branch. In the second step, the method realises only one iteration of geodesic dilation of the hole closing patch over the input object. An example showing the result of the dilation is presented in Fig. 2(c) where the intersection of a dilated hole closing patch and the input object is represented with white, shaded colour. In the following the intersection will be shortly called hole contour (see Fig. 2(d)). The third step consists in application of the ultimate homotopic skeleton algorithm [4] (UHSA) on the hole contour (see Fig. 2(e)). The last, fourth step consists in application of the HCA on the hole contour. As the hole contour does not contain any branch, the hole closing patch is not influenced by any branch and matches the geometry of the corresponding hole (see Fig. 2(f)).

This method, denoted by $\mathrm{HCA}+$, can be computed in quasi-linear time as UHSA has quasi-linear time complexity and all other steps have linear time complexity [14].

\section{Hole Filling Algorithm}

In this section we propose the original hole filling algorithm (HFA), which consists of 4th main stages and can be presented in the following general pseudocode:

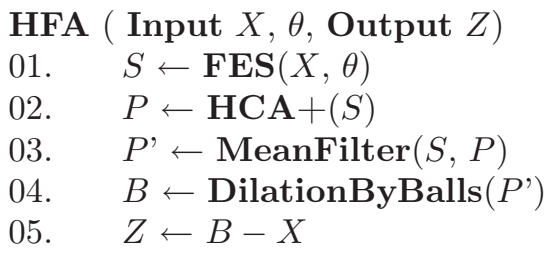

The first step of HFA consists in application of FES procedure which generates the filtered Euclidean skeleton of an input object $X \subset E$ originally proposed by Couprie, Coeurjolly and Zrour [5]. This state-of-the-art algorithm for skeleton generation is based on well defined mathematical notions and allows to gradually prune a generated skeleton which makes the HFA resistant to small noisy branches and deformations of an input object. The second step: $\mathbf{H C A}+$ procedure has been described in details in Sect. 4 Meanfilter is a simple procedure which realises propagation of an object thickness represented by values of its filtered skeletal voxels, into hole closing patches. The algorithm, in each iteration, calculates a new value for each voxel, from a hole closing patch, as an average value of voxels from its neighbourhood which belong either to the hole closing patch or to the filtered skeleton. The algorithm stops when no significant changes occur during an iteration.

The last procedure: DilationByBalls for each voxel $x$ of its input image generates a ball, centred in $x$, of radius equal to the value of voxel $x$.

Finally we obtain the hole filling algorithm which has the following properties: 
(a)

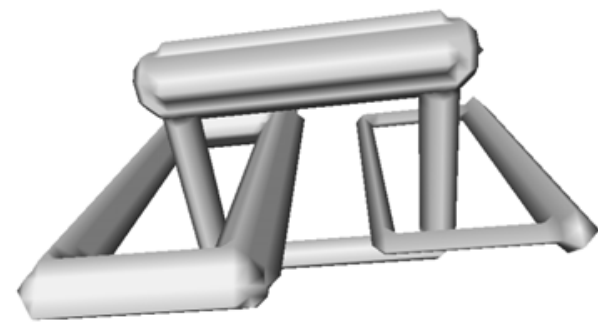

(b)

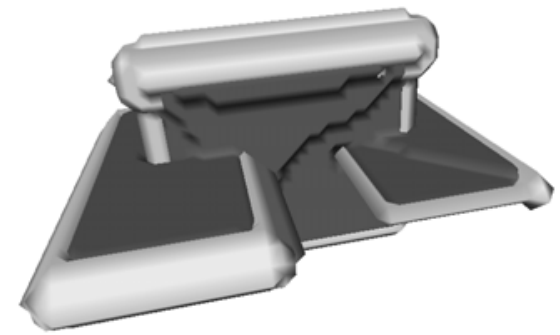

Fig. 3. Visualisation of a chain, whose links have different thicknesses. Hole filling volume is represented with dark grey colour: (a) an input object; (b) result of HFA applied to the chain.

- it is based on well defined mathematical notions and exact algorithms like: medial axis, bisector function, exact Euclidean distance, Euclidean skeleton,

- it generates volumes which fulfil both conditions 1 and 2 (see Sect. 3). The first one is guaranteed by FES and HCA + and the second is guaranteed thanks to MeanFilter and DilationByBalls,

- it is easy to use: only needs one parameter to be tuned (bisector threshold). Moreover it is easy to set the parameter as it belongs to the range $[0, \pi]$ and size of a hole closing patch changes monotonically with the bisector threshold. If the parameter is too small, then a skeleton of an input object is not enough pruned, hence noisy voxels from the surface of the object that could form cusp-like shapes may have influence on the hole filling volume which in that case is too thin. On the other hand, if the parameter is too large then an input object skeleton is over-pruned and the corresponding hole failing volume is too big and partly spread over the input object. Few tries are usually needed to set the proper bisector threshold for each input object.

- it is efficient: since most of its steps are optimal in time and space complexity.

Examples of the results of HCA are presented in Figs. 3, 4. In both examples, all holes are closed and the thickness of the corresponding hole filling volumes match the thickness of these objects, what is especially easy to observe in Fig. 3. So the two conditions about hole filling volume (see Sect. 3) are fulfilled. Figure 4 (d) presents an example cross-section of the filled crack from Fig 4(c). It can be observed that the thickness of hole, its filling volume, corresponds to thickness of the crack. Moreover cross-section of hole closing is presented with light grey colour in the figure. The cross-section is one voxel thick and it is "centered" in the crack. For a material science point of view, the hole closing has a microstructural meaning since it represents so-called bridge ligaments, i.e. small area of special grain boundaries which present high resistance to cracking. This phenomenon is usually met in sensitised stainless steel that can undergo intergranular stress corrosion cracking when subjected to special corrosive media [2]. In that case, if 
(a)

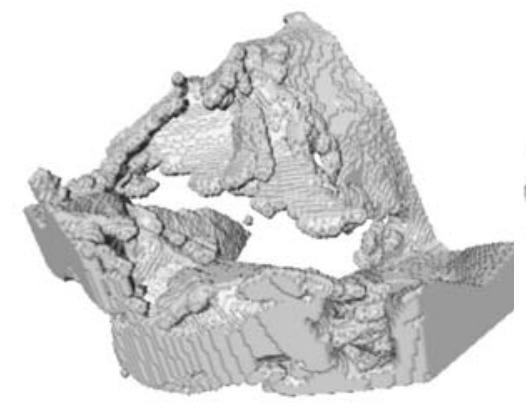

(c)

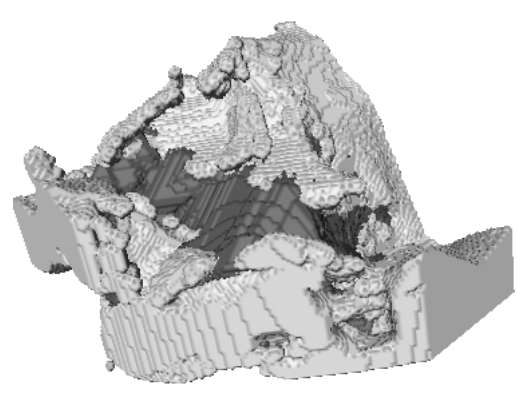

(b)

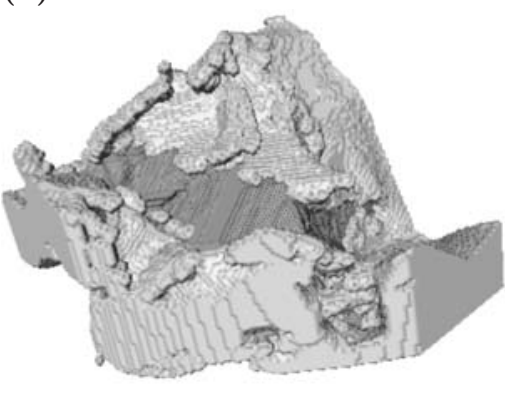

(d)

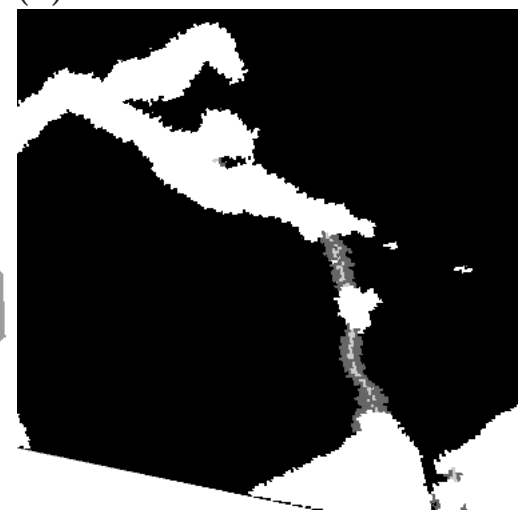

Fig. 4. A rendering of a crack path inside a material (material is represented by the background): (a) crack. Note that, there is a big hole inside the crack to be filled; (b) the crack with holes closed. Hole closing volume, one voxel thick, is represented with dark grey colour; (c) the crack with holes filled. Hole filling volume, which thickness corresponds to thickness of the crack, is represented with dark grey colour; (d) zoomed view of an oblique slice of the filled crack. Crack is represented with white colour, crosssection of hole filling volume is represented with dark grey colour and cross-section of hole closing volume is visualised with light grey colour.

a crack meets a bridge its branches go around the bridge and then merges. Hole filling has also a microstructural meaning since it is directly correlated to the local thickness of the crack portion that surrounds bridges. Work is currently in progress to correlate both morphological parameters of bridges, obtained after labelling of closed holes with local crack opening, retrieved from hole filling algorithm.

\section{Conclusions}

In the paper the authors have presented a flexible and efficient algorithm of hole filling for volumetric images. The algorithm has been tested on artificially constructed images and on images of a crack inside a material, for which it is an 
intended application. The visual analysis of results confirmed that the thickness of generated hole filling volumes correspond to the thickness of an input objects. According to our knowledge it is the first algorithm of hole filling for volumetric images.

Acknowledgements. The work is funded by the European Community's Sixth Framework Programme-Marie Curie Transfer of Knowledge Action (DENIDIA, contract No.: MTKD-CT-2006-039546). The work reflects only the author's views and the European Community is not liable for any use that may be made of the information contained therein.

The authors would like to thank Dr James Marrow (School of Materials, University of Manchester, UK) for permission to use volumetric images of crack.

\section{References}

1. Aktouf, Z., Bertrand, G., Perroton, L.: A three-dimensional holes closing algorithm. Pattern Recognit. Lett. 23, 523-531 (2002)

2. Babout, L., Marrow, T.J., Engelberg, D., Withers, P.J.: X-Ray microtomographic observation of intergranular stress corrosion cracking in sensitised austenitic stainless steel. Mater. Sci. Technol. 22, 1068-1075 (2006)

3. Bertrand, G.: Simple points, topological numbers and geodesic neighbourhoods in cubic grids. Pattern Recognit. Lett. 15, 1003-1011 (1994)

4. Bertrand, G., Couprie, M.: Transformations topologiques discretes. In: Coeurjolly, D., Montanvert, A., Chassery (eds.) Géométrie discrete et images numériques. J. M., Hermes, pp. 187-209 (2007)

5. Couprie, M., Coeurjolly, D., Zrour, R.: Discrete bisector function and Euclidean skeleton in 2D and 3D. Image and Vision Computing 25, 1543-1556 (2007)

6. King, A., Johnson, G., Engelberg, D., Ludwig, W., Marrow, J.: Observations of intergranular stress corrosion cracking in a grain-mapped polycrystal. Science 321(5887), 382-385 (2008)

7. Kong, T.Y., Rosenfeld, A.: Digital topology: introduction and survey. Comp. Vision, Graphics and Image Proc. 48, 357-393 (1989)

8. Park, W., Hoffman, E.A., Sonka, M.: Segmentation of intrathoracic airway trees: a fuzzy logic approach. IEEE Transactions on Medical Imaging 17, 489-497 (1998) 